\title{
Psychoanalysis and the brain - why did Freud abandon neuroscience?
}

\section{Georg Northoff*}

Mind, Brain Imaging and Neuroethics Research Unit, University of Ottawa Institute of Mental Health Research, Royal Ottawa Mental Health Centre, Ottawa, ON, Canada

Edited by:

Andrea Clarici, University of Trieste, Italy

Reviewed by:

Valentina Colonnello,

Albert-Ludwigs-University of Freiburg,

Germany

Antonio Alcaro, Università La

Sapienza di Roma, Italy

Claudio Colace, National Health

Service, Italy

*Correspondence:

Georg Northoff, Mind, Brain Imaging and Neuroethics Research Unit,

University of Ottawa Institute of

Mental Health Research, Royal

Ottawa Mental Health Centre,

Ottawa, ON, Canada.

e-mail: georg.northoff@theroyal.ca
Sigmund Freud, the founder of psychoanalysis, was initially a neuroscientist but abandoned neuroscience completely after he made a last attempt to link both in his writing, "Project of a Scientific Psychology," in 1895. The reasons for his subsequent disregard of the brain remain unclear though. I here argue that one central reason may be that the approach to the brain during his time was simply not appealing to Freud. More specifically, Freud was interested in revealing the psychological predispositions of psychodynamic processes. However, he was not so much focused on the actual psychological functions themselves which though were the prime focus of the neuroscience at his time and also in current Cognitive Neuroscience. Instead, he probably would have been more interested in the brain's resting state and its constitution of a spatiotemporal structure. I here assume that the resting state activity constitutes a statistically based virtual structure extending and linking the different discrete points in time and space within the brain. That in turn may serve as template, schemata, or grid for all subsequent neural processing during stimulus-induced activity. As such the resting state' spatiotemporal structure may serve as the neural predisposition of what Freud described as "psychological structure." Hence, Freud and also current neuropsychoanalysis may want to focus more on neural predispositions, the necessary non-sufficient conditions, rather than the neural correlates, i.e., sufficient, conditions of psychodynamic processes.

Keywords: Freud, psychoanalysis, neuroscience, brain, resting state

\section{THE SEARCH FOR THE LINKAGE BETWEEN PSYCHOANALYSIS AND NEUROSCIENCE - FREUD AND THE BRAIN}

Sigmund Freud - a neuroscientist? Often forgotten, Sigmund Freud, the founder of psychoanalysis, was trained initially as a neuroanatomist and investigated nerve cells and the exact lesion localization of speech disorders like aphasia. His early neuroscientific origins were though overshadowed and later even completely replaced by his focus on exclusively psychodynamic issues like the ego, our dreams, the unconscious. There is one specific node point in his own writings where his search for psychodynamic mechanisms converges with the quest for corresponding neuronal mechanisms in the brain. This is his famous 1895 writing on a "Project for a Scientific Psychology" where he tries to link neuronal mechanisms to psychodynamic concepts (see part two for details). Though he here attempts to link specific neuronal mechanisms to his psychodynamic concepts, Freud later considered his first neuropsychoanalytic endeavor a failure and consequently never did publish the manuscript during his life time (Freud, 1895).

After having its publication, Freud's "Project for a Scientific Psychology" has stirred much discussion about whether psychoanalysis can in principle be linked to neuroscience or not (see Brook, 1998; Levins, 2003; Peled, 2008; Northoff, 2011). Most recently, his 1895 Project writing has been taken as evidence to support the idea that psychoanalysis can indeed be linked to neuroscience which has led to the birth of a novel discipline, neuropsychoanalysis (see Weinstein and Kahn, 1956; Kandel, 1998; Solms et al., 1998; Solms and Solms-Kaplan, 2000; Solms and Turnbull, 2002; Fonagy, 2003; Schore, 2003; Mancia, 2004, 2006a,b; Solms, 2004; Northoff et al., 2007a; Feinberg, 2009; Northoff, 2011). Neuropsychoanalysis, most broadly defined, aims to link psychodynamic concepts and neuroscientific mechanisms and thus to integrate psyche and brain. However, as with any peculiar birth, the rather long pregnancy and consecutively painful delivery of the discipline of neuropsychoanalysis after its initial conception in 1895 has stirred much controversy; this concerns especially how the novel infant shall be properly taken care of with proponents and opponent being very much divided about the kind of remedy for the gestational complications.

Neuropsychoanalysis focuses predominantly on linking psychodynamic concepts like dreams, the unconscious, ego/self, to specific psychological, e.g., cognitive and affective functions, which in turn may be localized in particular brain regions (see below for further details). One focus has been on the unconscious and its relation to memories (see Kandel, 1998; Mancia, 2004, 2006a,b) while others searched for neuronal mechanisms underlying drives (Solms, 1996; Panksepp, 1998; Fonagy, 2003), dreams (Solms, 1997, 2000; Solms and Turnbull, 2002; Mancia, 2004; Hobson, 2009; Northoff, 2011), the ego (Northoff, 2007; Carhart-Harris and Friston, 2010), primary and secondary processes (CarhartHarris and Friston, 2010), and defense mechanisms (Fonagy, 2003; Northoff and Boeker, 2006; Northoff et al., 2007a; Feinberg, 2011; 
Northoff, 2011). Since these neuropsychoanalytic investigations focus on searching for the brain regions whose neural activity correlates with the psychodynamic concept in question, one may want to speak of the "neural correlates" of psychodynamic concepts (see below for details).

What would have Freud said to neuropsychoanalysis? Would he have embraced the newborn with the name neuropsychoanalysis? Or would he have left the novel infant alone in the very same way he rejected and abandoned his 1895 writing that, following him, was supposed to die a silent death in the graveyard of unpublished writings? We do not know and if at all we can only speculate. What we know though (at least partially) is why Freud abandoned his initial neuropsychoanalytic attempts. He considered the knowledge of the brain and thus the neuroscience at his time to be not as sufficiently mature as to allow for linking neuronal mechanisms and psychodynamic concepts.

How about the situation now? Is our current knowledge and insight into the brain's neuronal mechanisms sufficient to allow for what had eluded Freud, the link between neuronal mechanisms and psychodynamic concepts? Leading proponents like Solms (2004) and Panksepp (1998) do indeed consider neuropsychoanalysis as the continuation and completion of Freud's 1895 project (see also Pugh, 2006 as well as Ellenberger, 1970) and, more generally, of his endeavor to establish a scientifically based psychology of the human mind. Is neuropsychoanalysis thus nothing but a mere continuation and completion of Freud's aim to develop a scientific psychology of the mind which is now merely extended from the psyche to the brain? Let us turn finally to the brain itself and see whether neuropsychoanalysis can indeed overcome the deficits in the knowledge of the brain Freud diagnosed at his time.

Why did Freud abandon the brain and neuroscience completely after 1895? What we do know are the reasons Freud gave for abandoning his initial neuropsychoanalytic attempt, the "Project." He considered the neuroscience of his time to be insufficiently mature to allow for the linking of neuronal mechanisms and psychodynamic concepts. Besides the lack of knowledge, there may though be a more basic and principle reason namely how to approach the brain. Put in a nutshell, Freud's Project writing suggest an approach to the brain as an intrinsically active and dynamic organ rather than the brain being passive and static with its function being completely determined in a reflexive way. However, he could not find such a dynamic and active approach to the brain at this time and subsequently abandoned neuroscience. Instead, he dedicated himself completely to the psychic apparatus which he asked as active and dynamic when for instance developing his psychical topography, i.e., topological structure.

\section{THE SEARCH FOR NEUROPSYCHODYNAMICS - FUNCTION- AND LOCALIZATION-BASED APPROACH TO THE BRAIN}

Current neuropsychoanalysis heavily relies and draws on recent developments in Cognitive, Affective, or Social Neuroscience (see Panksepp, 1998; Gazzaniga et al., 2008). For instance, different cognitive functions like attention, working memory, episodic memory, are taken as initial starting points and are then related to supposedly corresponding concepts like memory or dreams within the psychodynamic context (see for instance, CarhartHarris and Friston, 2010, and Mancia, 2006a,b for such an approach). While others like Panksepp and Solms pursue a more affective-based approach to neuropsychoanalysis that relies on the intrinsic affective functions and their corresponding subcortical neural substrates (Panksepp, 1998; Solms and Turnbull, 2002). This has lead to the discussion of the neuronal mechanisms underlying psychodynamic concepts like introjections, narcissism, self-objects, drives (see Solms, 2004; Northoff et al., 2007a; Northoff and Panksepp, 2008; Northoff, 2011). One may consecutively want to speak here of what I call a "function-based approach" as the first hallmark of current neuropsychoanalytic approaches.

How can such function-based approach be related to the brain? Broadly speaking, Cognitive, Affective, or Social Neuroscience aim to link cognitive, affective, or social psychological functions to the brain and more specifically to the neural activity in specific regions of the brain. For instance, cognitive functions like working memory, attention, have been associated with the neural activity in specific cortical regions like the lateral prefrontal cortex and the parietal cortex (Gazzaniga et al., 2008). While affective and basic social functions are more associated with neural activity in subcortical regions like the tectum, the periaqueductal gray (PAG), the dorsomedial thalamus, the colliculi (see Damasio, 1995, 2000; Panksepp, 1998; Panksepp and Northoff, 2009). Following the roadmaps of its guiding disciplines, Neuropsychoanalysis aims to link specific psychodynamic mechanisms to the neuronal activity in particular regions of the brain.

One of the main frontrunners of such approach was Mark Solms. He observed particular psychodynamic changes in his neurosurgical patients who suffered from specific lesions in their brains. Inferring from the pathological to the healthy, this allowed him to link psychodynamic mechanisms to specific brain regions (Solms and Solms-Kaplan, 2000; Solms and Turnbull, 2002) and to consecutively establish the discipline of neuropsychoanalysis. Relying on his observations in neurosurgical patients, he for instance argues that the ventromedial prefrontal cortex (VMPFC) may be crucial in constituting the ego (or self; see chapter three in part three for detailed discussion) while the parietal cortex may be central in constituting the own body as first self-object (see Solms, 1999). This approach has recently been extended also to the healthy brain so that neuropsychoanalysis can be characterized by what I call a "localization-based approach" as it is the second hallmark feature.

Does neuropsychoanalysis in this sense, as characterized by the two hallmark features of a function- and localization-based approach, can account for what had eluded Freud in his early 1895 writing? Can the function- and localization-based approach to the brain in current neuropsychoanalysis make up for the deficits in our knowledge of the brain that Freud diagnosed at his time? Would such a function- and localization-based approach have led Freud to reverse his decision to abandon and abort his 1895 writing? To decide these questions, we have to turn back to Freud himself and see how he would have approached the brain if he only could.

\section{THE SEARCH FOR THE PSYCHIC APPARATUS - PSYCHOLOGICAL STRUCTURE AND ORGANIZATION}

How did Freud approach psychological functions? How did he relate them to what he called the "psychic apparatus?" Freud 
listened to his patients and investigated their mental contents which he then associated with specific psychological functions. The psychological functions in turn were assumed to mirror a specific psychodynamic mechanism. For instance, the dreams showed his patient's mental contents to be predominantly sexual which otherwise, outside dreams, could not surface. From that he inferred a specific psychological mechanism, repression, that repressed sexual contents during the day but let them surface in the night's dreams.

What does Freud presuppose here? He starts with observations of mental contents as for instance sexual desire and attributes a specific meaning to them in that they are supposed to express something specific about the person itself. Now the crucial step comes. He infers from the mental content and its personal relevance a specific psychological function, repression. What does he presuppose here? He presupposes that the mental content and its specific meaning are possible only on the basis of the specific psychological function of repression. One may consequently want to speak of a "function-based approach" in Freud.

Is there any difference between the "function-based approach" in the context of Cognitive, Affective and Social Neuroscience and the one presupposed by Freud? In contrast to Freud, Cognitive, Affective and Social Neuroscience do not start with specific individually meaningful contents that are subjective. Instead, they start with certain objective behaviors as for instance social interaction, cognitions, or emotions. Despite such difference in the starting point, the inference to psychological functions is the same. In the same way that Cognitive, Social, and Affective Neuroscience infer specific psychological functions to account for the observed behavior, Freud infers his psychological functions from the mental contents he observed. One may consequently speak in both cases of a "function-based approach" (see below for a critical discussion though).

There is even further convergence. Many of the cognitive, affective, and social functions investigated in Cognitive, Affective, and Social Neuroscience are related these days to the psychological functions Freud described. For instance, the cognitive function of memory and especially autobiographical memory may be closely related to Freud's psychological function of repression (Kandel, 1998; Mancia, 2006a,b). Freud's "function-based approach" may consecutively converge with the one presupposed in current Cognitive, Affective, and Social Neuroscience.

Does convergence of both function-based approaches provide a common platform to make the second step, the one to the brain? If the cognitive, affective, and social functions can be localized in specific regions of the brain, the respectively associated psychological functions as described by Freud must be related to exactly these brain regions. The "localization-based approach" would then be seen as the natural extension of Freud's psychological functions to the brain. This is, as indicated above, the way Mark Solms and Jaak Panksepp see it.

However, Freud did not go this way. He did not extend and complement his function-based approach by a localization-based approach. Why? Let us listen to Freud himself: "Every attempt to discover a localization of mental processes. . . has miscarried completely. The same fate would await any theory that attempted to recognize the anatomical position of the system (consciousness) as being in the cortex, and to localize the unconscious processes in the subcortical parts of the brain. There is a hiatus which at present cannot be filled, nor is it one of the tasks of psychology to fill it. Our psychical topography has for the present nothing to do with anatomy." (Freud, 1915)

Why was Freud so skeptical about the localization of his psychological functions? Is it only because he lacked the psychological inventory of Cognitive, Affective, and Social Neuroscience we are fortunate to have these days? This is the position Mark Solms, Jaak Panksepp, and many proponents of Neuropsychoanalysis take. But let us go back to Freud himself and see how his focus shifted especially after his 1895 writing.

After his 1895 writing, Freud abandoned the reference to the brain completely and focused exclusively on psychological functions. He however went one step further beyond mere psychological functions. He aimed to put the assumed psychological functions into a larger psychological context. This context refers to psychological structure and organization rather than to specific psychological contents as yielded by particular psychological contents. For instance, he introduced the tripartite structure of the psychic apparatus with its division between Id, Ego, and Superego. His focus thus goes beyond mere psychological functions to their underlying psychological structure and organization. Unlike Cognitive, Affective, and Social Neuroscience, he does not complement his function-based approach by a localization-based approach. Instead, he here departs taking another direction, the one of psychological structure and organization. I consecutively speak of what I call "structure-based approach" in Freud.

How are psychological structure and organization related to psychological functions? Psychological structure and organization enable and predispose specific psychological functions as for instance the ego makes possible the repression of sexual desire. Let us describe the difference between psychological functions and structure/organization in the terms of necessary and sufficient conditions. Psychological functions are assumed to correlate with the mental contents (or the behavior) in question; the former is thus a sufficient condition of the latter. This is different in the case of psychological structure and organization. The ego is not assumed to correlate with specific mental contents and can therefore not be regarded a sufficient condition. However, it is assumed to make first and foremost possible all kinds of mental contents. Without ego, there can be no mental contents at all. The ego is thus a necessary but non-sufficient condition of (possible) mental contents.

Where does this leave us? Freud and current Cognitive, Affective, and Social Neuroscience seem to share the function-based approach. While sharing the first step, they though depart in the subsequent step. Cognitive, Affective, and Social Neuroscience go forward toward the brain when assuming a localization-based approach. Freud though decided to go into another direction, the direction of psychological structure and organization thereby presupposing a structure-based approach. If we want to extend Freud to the brain, we thus have to extend his structure-based approach into a neural context. For that we may leave Freud and go back to the neuroscience at his and our time. 
We have to be careful though. Freud did already pursue a structure-based approach before he abandoned neuroscience and switched completely to psychology and psychoanalysis. This is well apparent in his early structural distinction between preconscious, unconscious, and conscious. One may want to read his 1895 writing on the brain thus a the attempt to find such structure in the brain itself. This may well correspond to his search and postulation of the kind of dynamic mechanisms he suggested in his 1895 writing. Due to the limited knowledge of the brain at his time, he may have felt it to be hopeless to ever link his structural approach on the psychological level to corresponding neuronal structure within the context of the brain. That though is mere speculation. I therefore refrain from that and characterize our current knowledge about the brain and its neuronal structure.

\section{THE SEARCH FOR THE BRAIN - INTRINSIC VERSUS EXTRINSIC VIEWS}

Freud focused on the structure and organization of the psychic apparatus, i.e., its psychological structure and organization. How can we now put what he described as psychological structure and organization into the neural context of the brain? One may be inclined to argue that we simply have to look for what may be described as "neural structure" of the brain itself. How can we find the brain's neural structure? Apparently it does not seem to consist in the various psychological functions and their respectively associated regions investigated in current neuroscience. Analogous to the psychic apparatus in Freud's case, the brain itself may show an intrinsic neural structure independent of the kind of extrinsic information, i.e., stimuli associated with particular events or objects, processed in the brain's various regions and their respective functions. This shifts the focus to the intrinsic activity of the brain, what is often called resting state activity, and the kind of neural structure it constitutes.

At the beginning of neuroscience around the turn of the twentieth century, different views of the brain emerged. One view favored by the British neurologist Sir Charles Sherrington (1857-1952) assumed the brain and more specifically the spinal cord to be primarily reflexive. Reflexive means that the brain reacts in predefined and automatic ways to stimuli from the outside world. Hence, the extrinsic stimuli do almost completely and exclusively determine the activity in the brain which is thus driven by the momentary demands of the extrinsic environment, i.e., the sensory inputs. This means that what we as observers call "stimulus-induced activity" can be completely and exclusively be traced back to the stimulus itself implying, to put it in more general terms, that the "brain has no say in what happens in the brain." I call such a view of the brain an "extrinsic view" because the brain is here not characterized by intrinsic features that by themselves determine what can (and cannot) occur in the brain (see Figure 1A).

An alternative view though was suggested by one of his students, T. Graham Brown. In contrast to his teacher, he suggested that the brain's activity, i.e., spinal cord and brain stem, is not primarily driven by extrinsic stimuli from the environment but rather by intrinsic activity within the spinal cord and the brain stem itself. This means that stimulus-induced activity can no longer be completely and exclusively be traced back to the stimulus itself. Instead, what we as observers describe as stimulus-induced activity must
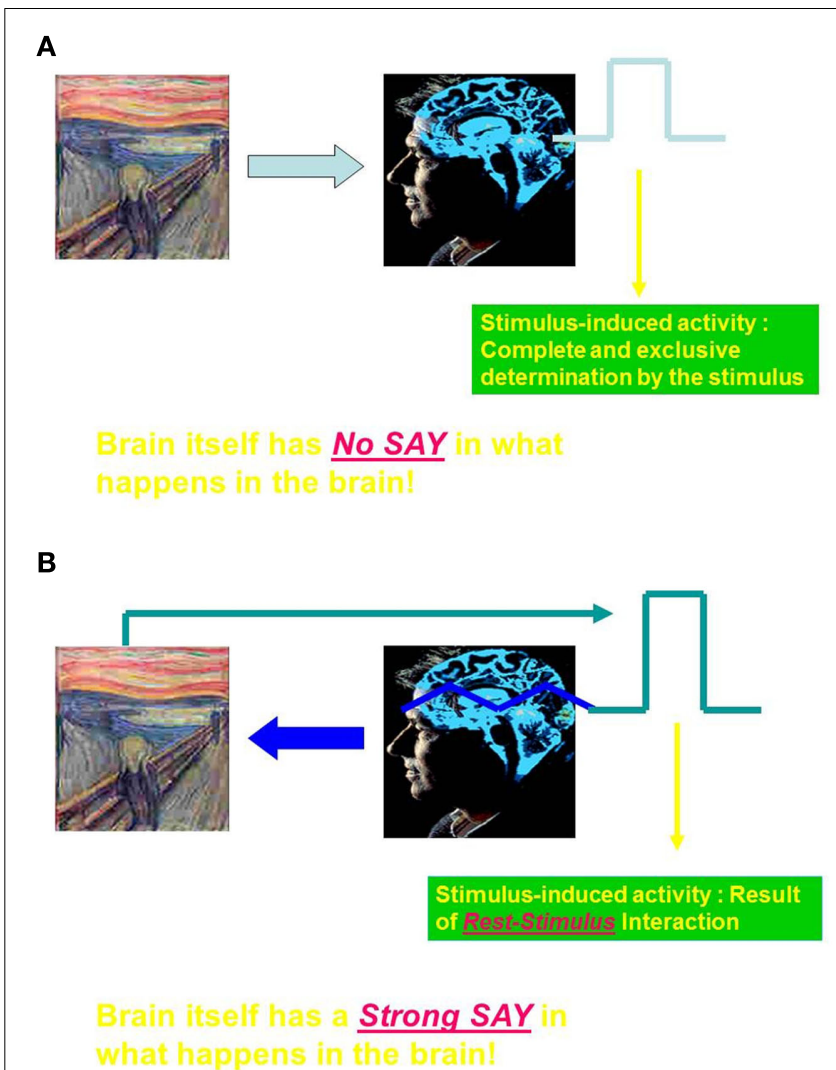

FIGURE 1 | (A) Extrinsic view- brain as behavioral-cognitive reflex apparatus (Sherrington, cognitive neuroscience). (B) Intrinsic view- brain as active player in its neuronal activity (Brown, Lashley, Llinas, Shulman, Panksepp).

then be considered to be the hybrid result of a specific interaction between the intrinsic activity and the stimulus. Hence, unlike the extrinsic view of the brain, the brain itself and more specifically its intrinsic features have a say in what happens in the brain I therefore speak here of an "intrinsic view" of the brain (see Figure 1B).

The dichotomy between an intrinsic and an extrinsic view of the brain is preserved into our times and has recently, within the context of functional brain imaging, seen a resurgence (see Raichle, 2009a). Let us start with the extrinsic view first which is most predominant in especially cognitive and social neuroscience. Cognitive and social neuroscience employ specific experimental tasks and associated stimuli to probe for neural activity changes within the brain. The extrinsic cognitive stimuli can thereby be related to neural activity in the brain. This has led to the view of a tight relationship between the brain's neural activity and the extrinsic stimulus with the latter exclusively and sufficiently determining the former.

Sherrington's view of the brain as primarily reflexive with regard to sensory and motor functions is here extended into the domain of cognitive, affective, and social functions. Rather than responding to sensory stimuli as in the case of Sherrington, the brain now passively and automatically reacts in an almost reflexive way to cognitive stimuli and demands from the environment. One 
may consequently want to speak of an extrinsic and cognitive view of the brain. Hence, cognitive neuroscience and its more recent siblings of affective and social neuroscience, all strongly relying on functional imaging, thus presuppose an extrinsic rather than an intrinsic view of the brain.

This extrinsic and cognitive view of the brain has however been challenged recently by results from functional imaging itself which has been the main tool to advocate the extrinsic approach to the brain in cognitive neuroscience. Studies by Raichle et al. (2001) showed that the brain is not only active when we stimulate it with our extrinsic stimuli and observe the associated activity changes, so-called stimulus-induced activity. Even when we rest without any specific extrinsic stimulation, our brain is still active and shows high resting state activity. This is for instance reflected in the fact that our brain is energy-hungry even in the resting state. Though comprising only $2 \%$ of the body's mass, the resting awake brain consumes $20 \%$ of the body's total oxygen. Oxygen is essential for any kind of neuronal activity and the abundant use of the body's oxygen during rest indicates that something must go in the brain during rest (Raichle, 2009a,b).

What does the brain do with the huge amount of energy ( $20 \%$ of the body's energy) it apparently invests into its intrinsic activity? Part of this intrinsic energy the brain uses to process extrinsic stimuli. For that though the brain spends only a tiny fraction, around $2-10 \%$, of its total energy budget. What happens to the rest of the energy? This raises the question for the use and purpose of the intrinsic activity that cannot consist in mere stimulus processing since then a higher amount of energy would be invested into it. What does the brain do with its intrinsic activity into which it spends so much energy? We do not know yet. One may consider the brain's high resting state activity as mere noise in the background of stimulus-induced activity with the latter being the real thing. But why then does the brain waste so much energy and effort for mere noise? There must be more to the brain's resting state activity. This "more" remains unclear though.

\section{THE SEARCH FOR THE BRAIN'S RESTING STATE - SPATIAL AND TEMPORAL FEATURES}

How can we describe the brain's resting state activity in further detail? Besides the high metabolism, the resting state activity can be characterized by strong especially low frequency fluctuations in the range between 0.1 and $0.0001 \mathrm{~Hz}$ (see Raichle et al., 2001; He et al., 2008; He and Raichle, 2009). While higher frequency fluctuations $(1-60 \mathrm{~Hz})$ are certainly present in the resting state too, they though become more accentuated during subsequent stimulus-induced activity.

There is abundant evidence for intrinsic activity in the brain that goes on continuously so that the brain is never really "at rest" but shows continuously high activity. The term resting state is consecutively used in a more operational and experimental context where it is determined as the absence of specific stimuli from the outside of the brain (see Eijsden et al., 2009; Logothetis et al., 2009; Shulman et al., 2009; Northoff et al., 2011; Raichle, 2010; see Appendix for more details on the conceptual issues). This is the meaning of the term "resting state" that shall be used in the following.
Let us look at the brain's resting state in more detail. High resting state activity has been observed though not only on the cellular or microscopic level of the brain but also on a macroscopic level, e.g., on the level of macroscopically identifiable regions. Early studies in humans using PET identified high oxygen and glucose consumption in the resting state in a particular set of regions including anterior and posterior cortical midline regions like the VMPFC, the dorsomedial prefrontal cortex (DMPFC), the different parts (sub-, pre-, and supragenual) of the anterior cingulate cortex (ACC), the posterior cingulate cortex (PCC), and the precuneus, as well as other regions like the lateral parietal cortex and the hippocampus (see Raichle et al., 2001; Simpson et al., 2001; Greicius et al., 2004). These regions have consequently been subsumed under the concept of the default-mode network (DMN) that include what has been called the cortical midline structures (CMS; Raichle et al., 2001, see Buckner et al., 2008 and Broyd et al., 2009 for recent reviews; see though Morcom and Flher, 2007 for a critical view, Northoff et al., 2006).

Analogous observations were made in fMRI. During presentation of external stimuli as for instance emotional or cognitive tasks, these regions show predominantly negative signal changes in fMRI, deactivation, or negative BOLD response (NBR) in their neural activity whose underlying physiological mechanisms remain unclear though. The regions showing such deactivation or NBR during stimulation must be distinguished from those that show activation or positive BOLD responses (PBR) in AMRI. This has let to the distinction between task-positive regions and task-negative regions (see below for details). While being deactivated during stimulus- or task-induced activity, the regions of the DMN show high activity and a high degree of intrinsic functional connectivity in the resting state (Greicius et al., 2004; Beckmann et al., 2005; Fox et al., 2005; Fransson, 2005; Damoiseaux et al., 2006; Buckner et al., 2008). At the same time, the task-negative regions of the DMN as linked by high intrinsic connectivity show PBR and are negatively correlated, e.g., anticorrelated with the task-positive regions during the resting state. Therefore, taskpositive and task-negative regions are not only anatomically distinguished but also functionally, i.e., in their BOLD responses and connectivity.

Another feature of the brain's intrinsic activity is spontaneous fluctuations in neural activity. Using electrophysiological recordings such as EEG, Llinas (1988) and others (Arieli et al., 1996; Buzsaki and Draguhn, 2004; Buzsaki, 2006) have observed intrinsic brain activity in the gestalt of auto-rhythmic electrical oscillations (or synchronizations) across different brain regions, for instance the thalamic nuclei and cortical regions.

Spontaneous signal fluctuations in rather low frequency ranges of the BOLD signal can be observed using fMRI (rather than EEG). The spontaneous BOLD fluctuations are to be found in lower frequency ranges including the delta band $(1-4 \mathrm{~Hz})$, up- and-down states $(0.8 \mathrm{~Hz})$ and infra-slow fluctuations (ISF's; $0.01-0.1 \mathrm{~Hz}$ ). All three, delta, up-and-down states and ISF's, are often subsumed under the concept of slow cortical potentials (SCP) as they can be measured with EEG (with a special amplifier needed though). These SCP seem to be related to the spontaneous BOLD fluctuations (He et al., 2008; Khader et al., 2008) with both representing fluctuations in cortical excitability across time. This in turn may 
affect the spiking activity of neurons on the cellular level as well as activity changes on the regional level. However, it remains unclear how spontaneous cellular activity on the level of neurons translates into the spontaneous fluctuations in (and across) regions during the resting state (see Figure 2).

Spontaneous fluctuations of neural activity as an indicator of intrinsic activity have been observed in the regions of the DMN, e.g., so-called task-negative regions, but also in other taskpositive regions including sensory cortices, motor cortex, insula, and subcortical regions mirroring so-called task-positive regions (see Freeman, 2003; Shulman et al., 2004, 2009; Hunter et al., 2006; Wang et al., 2007; Buckner et al., 2008). Further support for spontaneous resting state activity across the whole brain comes from electrophysiological studies showing spontaneous neuronal oscillations and synchronizations in various parts of the brain including the hippocampus, the visual cortex (Llinas, 1988; Arieli et al., 1996; Singer, 1999; Fries et al., 2001, 2007; Nase et al., 2003; Buzsaki and Draguhn, 2004; Buzsaki, 2006). This suggests that spontaneous fluctuations and thus intrinsic activity may be prevalent throughout the whole brain in both humans and animals and not be limited to the DMN.

What does the brain do in resting state? Is there a hidden light as for instance a mind within the brain that consumes all that energy and activity? No there is nothing but a bunch of neurons in our brain. But these neurons themselves seem to use a lot of energy themselves. They show high activity as high firing rates with various spikes per second already in the resting state. This suggests that our neurons are always active, there are never at rest; even if we feel that we do not do anything and enjoy our rest, our brain does not seem to have the luxury of a true rest. Unlike a car whose engine we turn of once we no longer want to drive, the brain is continuously active. The brain is an engine that never stops working and, unlike the car and its engine, keeps us continuously driving which can be observed even in sleep as for instance when we are dreaming. We though have to be clear that currently the exact purpose of the brain's resting state remains unclear. Whether it serves some adaptive purpose may be open to discussion. Moreover the neural coding, the coding of neural activity in the resting state remains far from clear (see Northoff, 2012).

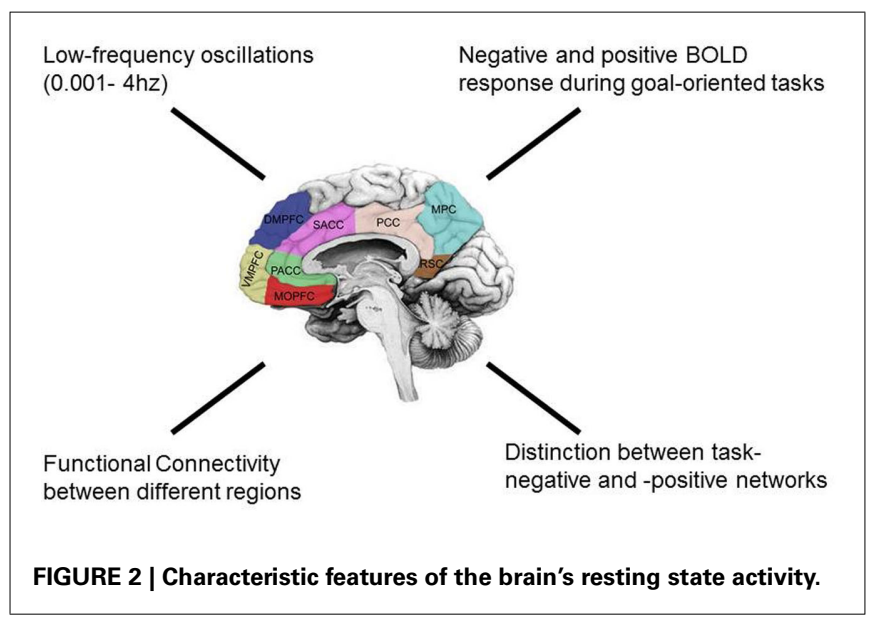

\section{THE SEARCH FOR CORRESPONDENCE - THE RESTING STATE'S SPATIOTEMPORAL STRUCTURE AND THE PSYCHOLOGICAL STRUCTURE OF THE PSYCHIC APPARATUS}

I demonstrated that the brain's resting state activity can be characterized by particular temporal, i.e., low frequency fluctuations, and spatial, i.e., functional connectivity, patterns. Taken together, this yields a certain spatial and temporal structure, a "spatiotemporal structure" as I name it in the following. The term spatiotemporal structure comes close to what Fingelkurts et al. (2010) describes as "operational space time" (OST) which he assumes to be yielded by the brain's resting state.

What does this spatiotemporal structure mean in more specific terms? The brain's resting state's spatiotemporal structure must be regarded as a structure that is based upon the input of all three stimuli, neuronal and intero- and exteroceptive stimuli, and their respective spatiotemporal structures, i.e., their statistical distributions across time and space. If they are matched and compared with each other, the resulting spatiotemporal structure must span across brain, body, and environment thus constituting what may be described a "virtual spatiotemporal structure." Since the brain's resting state activity continuously interacts with intero- and exteroceptive stimuli based on either past or actual stimulus-rest interactions, the latter are integrated into the brain's resting state activity thereby constituting such virtual spatiotemporal structure.

In order to constitute such virtual spatiotemporal structure, the brain bestows and imposes it upon all the stimuli it processes, be they of neuronal, intero-, or exteroceptive origin. As such it provides a grid, template, or schemata along whose lines all neural activity is organized and structured. The need to assume such template or schemata has already been nicely described by K. Lashley: "A second point of major importance is that the nervous system is not a neutral medium on which learning imposes any form of organization whatsoever. On the contrary, it has definite predilections for certain forms of organization and imposes these upon the sensory impulses which reach it. ... In its functional organization the nervous system seems to consist of schemata or basic patterns within which new stimuli are fitted.” (Lashley, 1949, p. 35)

Finally, we may want to briefly go into a conceptual issue. What exactly is meant by the concept of "spatiotemporal structure?" When I am talking of a spatiotemporal structure, I am not talking of real space and time as measured and observed in physics. I am rather talking of spatiotemporal structure in a virtual sense that as such cannot be measured and observed objectively as time and space in physics. This implies also that the spatiotemporal structure cannot be considered a mere simulation and consecutively a representation of the physically and objectively measurable time and space of the world.

A related issue is the exact characterization of neuronal mechanisms that are supposed to underlie what is described in empirical concepts like functional connectivity and low frequency fluctuations. The empirical concepts of functional connectivity and low frequency fluctuations do not describe physiological mechanisms but rather statistical features (see also Fingelkurts, 2004; Fingelkurts and Kahkonen, 2005 for the discussion of this issue). More specifically, functional connectivity describes the statistically based temporal co-variation of neural activity between different regions 
and thus across space while low frequency fluctuations concern the statistical variation of neural activity across time. In short, both empirical concepts of functional connectivity and low frequency fluctuations must be assumed to describe statistical rather than physiological features. As such they must be regarded as synthetic and virtual as the spatiotemporal structure they constitute.

I characterized the brain's resting state activity by a spatiotemporal structure which is not real in physical terms but rather statistically based, synthetic and virtual. Due to the overlap with regard to the term "structure," one may now be inclined to compare what Freud described as "psychological structure" of the psychic apparatus with what I here illustrated as the brain's neural structure.

There is certainly a similarity in that both psychological structure and neural structure are not real in the physical sense but rather virtual. And both share that they are synthetic meaning that they are the result or product of a process that constitutes them (with the exact nature of this constitutional process remaining unclear though). Both concepts are also analogous in that they do not target physical or psychological properties, i.e., entities but rather a particular structure and organization. Interestingly, Freud uses indeed the spatialization of his psychological structure when he speak of the topological structure of the ego. He thus uses the spatial dimension to characterize his psychological structure. In the case of the brain, such spatial and temporal structure is even more direct, and, to be more precise, the brain's neural structure is by itself defined in spatial and temporal terms.

In sum, one may assume correspondence between Freud's approach to the psychic apparatus in terms of psychological structure and the view on the brain and its resting state's intrinsic spatiotemporal structure. The question is now whether and if so how both are related to each other since that would provide an answer to whether Freud would embrace neuropsychoanalysis and why he abandoned neuroscience at this time.

\section{THE SEARCH FOR NECESSARY AND SUFFICIENT CONDITIONS - “NEURAL CORRELATES” VERSUS “NEURAL PREDISPOSITIONS"}

Let me venture briefly into current neuroscience. One of the main fields in neuroscience these days is the search for the neuronal mechanisms underlying consciousness. This is described and subsumed under the heading of neural correlates of consciousness (NCC; Koch, 2004). The NCC aims to identify the neuronal mechanisms that are sufficient to induce consciousness. Thereby it aims to describe the relationship of consciousness to specific psychological functions like attention, working memory, as well as how consciousness is related to the neural activity in specific brain regions (see Koch, 2004; Northoff, 2011). The function- and localization-based approach is thus well prevalent in the NCC (though admittedly in a somehow modified form; see Northoff, 2011 for details).

Analogously to the case of consciousness and the NCC, current neuropsychoanalysis also aims to search for the sufficient neural conditions of specific psychodynamic mechanisms. This is for instance the case when Solms locates early defense mechanisms like introjections and other forms of internalization with the region of the VMPFC. Hence, analogous to the NCC, one may want to speak of what I call "neural correlates of psychodynamics"
(NCP). The NCP shares with the NCC the focus on the sufficient neural conditions as well as (the rather implicit presupposition of) a function- and localization-based approach. There is one crucial difference though. Instead of being restricted to consciousness as the NCC, neuropsychoanalysis targets both conscious and unconscious states. The NCP do consequently cover a more extensive territory, e.g., consciousness and unconsciousness, when compared to the NCC and its exclusive focus on consciousness.

The NCP focus on the sufficient neural conditions of the psychological functions Freud described. How about their necessary neural conditions? We recall. Freud extended and complemented psychological functions by his search for psychological structure and organization and thus by a structure-based approach. Instead of sufficient conditions, psychological structure and organization concern the necessary but non-sufficient conditions of mental contents. How can we extend the psychological structure and organization to the brain? The mere neural correlates, the NCP, are not appropriate since they refer only to the sufficient but not the necessary conditions. We instead need to target those neural conditions that are necessary but non-sufficient by themselves. Since those neural conditions may enable and predispose the psychological functions and mental contents, I here speak of "neural predisposition."

Let us determine the concept of neural predispositions in more detail. The concept of neural predispositions describes the necessary but non-sufficient conditions provided by the brain itself; these conditions are supposed to enable and predispose psychological functions and their respective mental contents. One may consequently want to speak of what I call "neural predispositions of psychodynamics" (NPP) that refer to the necessary but nonsufficient, e.g., enabling and predisposing neural conditions of mental contents as described in psychodynamic concepts. As such the NPP must be distinguished from the NCP that refer to the sufficient but non-necessary, e.g., operating and executing, conditions of the mental contents as referred to in psychodynamic concepts.

After having charted out the conceptual territory, we now finally can turn to the empirical grounds of the brain. What do neural predispositions and thus NPP refer to in terms of empirical brain function? While the neural correlates and the NCP as being function- and localization-based can be associated with the neural activity in specific brain regions, this remains unclear so far in the case of the neural predispositions. I suggest that for that we have to go back to the brain itself and its intrinsic features and more specifically to its resting state activity and its spatiotemporal structure.

What does the brain do with this high level of resting state activity? One may consider the brain's high resting state activity as mere noise in the background of stimulus-induced activity with the latter being the real thing. But why then does the brain waste so much energy and effort for mere noise? There must be more to the brain's resting state activity. This "more" remains unclear though currently. If there is "more" to the brain's resting state activity than mere noise, it must somehow affect stimulus-induced activity with the latter being dependent upon the former. Hence there must be what in the following I will call "rest-stimulus interaction" (see Northoff et al., 2010 for an overview). While the exact neuronal 
mechanisms making such rest-stimulus interaction possible are unclear, there is at least empirical evidence for such interaction.

Recent studies do indeed lend empirical support to such reststimulus interaction. Maandag et al. (2007) for instance manipulated the resting state activity level in rats with anesthetic drugs and investigated their neural activity changes during subsequent execution of similar movements. The different resting state levels lead to different neuronal activity patterns in the cortex during the subsequent movement with some regions being active only during a specific resting state activity level and others being recruited in another one. Human studies showed that the degree of stimulus-induced activity in the perigenual anterior cingulate and the visual cortex is dependent upon the resting state concentration of GABA in the very same regions (Northoff et al., 2007b; Muthukumaraswamy et al., 2009; see also Boly et al., 2007 for another example and Northoff, 2011 for a review).

In addition to such rest-stimulus interaction, there is also reverse traffic with stimulus-induced activity impacting and modulating subsequent resting state activity amounting to what I call "stimulus-rest interaction." Lewis et al. (2009) for instance demonstrated that visuospatial learning did not only lead to activation changes in the visual cortex but also to connectivity changes of that region with DMN regions during the subsequent resting state (See Northoff et al., 2010 for review). Similar observations were made in studies on working memory and self-relatedness. Higher degrees of working memory and self-relatedness lead to stronger connectivity and activity changes within the DMN in subsequent resting state periods when compared to lower degrees of working memory and self-relatedness (Schneider et al., 2008; Pyka et al., 2009).

What do these examples tell us about the brain and its involvement in specific cognitive, affective or social functions as described by particular psychodynamic concepts and localized in specific brain regions? When investigating the brain's resting state activity and how it impacts stimulus-induced activity (and consequently psychological functions; see also Northoff et al., 2010 for a recent review), we focus on the necessary but non-sufficient conditions and thus on those neural conditions that enable and predispose psychological functions and their respectively associated mental contents. One may then describe the brain's resting state activity and its impact on subsequent stimulus-induced activity as necessary but non-sufficient conditions and hence as enabling and predisposing rather than executing and operating conditions. In other terms, the investigation of the brain's resting state activity may give us a glimpse into what I above described as "neural predisposition" as distinguished from mere neural correlates. We though have to be clear that currently we do not know the exact neuronal mechanisms that may predispose the resting state and its spatiotemporal structure to generate consciousness (see Northoff, 2012).

\section{THE SEARCH FOR THE LINKAGE - “NEURAL PREDISPOSITIONS" AS NECESSARY CONDITION OF "PSYCHOLOGICAL PREDISPOSITIONS"}

How does all that relate to Freud? In the same way that the brain's resting state activity is a neural predisposition for subsequent stimulus-induced activity, Freud assumes that the ego's psychological structure provides the psychological predisposition for various psychological functions ranging from sexual drive to memory. Hence, Freud was very much interested in what may be described as "psychological predispositions," the necessary but non-sufficient conditions of psychological functions.

This raises the question how both neural and psychological predispositions are related to each other. One may now be inclined to argue that the resting state's spatiotemporal structure may be central and thus necessary and predispositional for constituting what Freud described as psychological structure. This would mean to link Freud's psychological structure to the spatial and temporal features characterizing the resting state's neural structure. While this is certainly a research project for the future, it would make possible an intrinsic linkage between neural and psychological structures and ultimately between brain and psychic apparatus and hence between neuroscience and psychoanalysis (see Figure 3).

Let us get back to our starting point. We were asking why Freud abandoned neuroscience after his 1895 Project writing and shifted from the brain to the psychic apparatus. Based on his primary interest in the psychological structure and organization of the psychic apparatus, he could not find any analogous approach to the brain at this time. All he could find was a focus on particular regions and functions and thus a region- and function-based approach to the brain. This amounted to a rather extrinsic view on the brain that focused more on how stimuli are processed in the brain rather than on the brain itself and its intrinsic features independent of the stimuli. Hence, he remained unable to detect and see the brain's intrinsic neural structure which would have complemented his search for the psychological structure within the neural context of the brain. Rather than searching for something that, for him at his time, did not seem to exist, he preferred to develop the psychological structure in more detail thus venturing into psychoanalysis while abandoning neuroscience.

What can we learn from this today? Besides the extrinsic approach to the brain, we should also develop a more intrinsic view on the brain and its intrinsic features. This will allow us to reveal the brain's neural structure which I here assumed to be a "virtual"

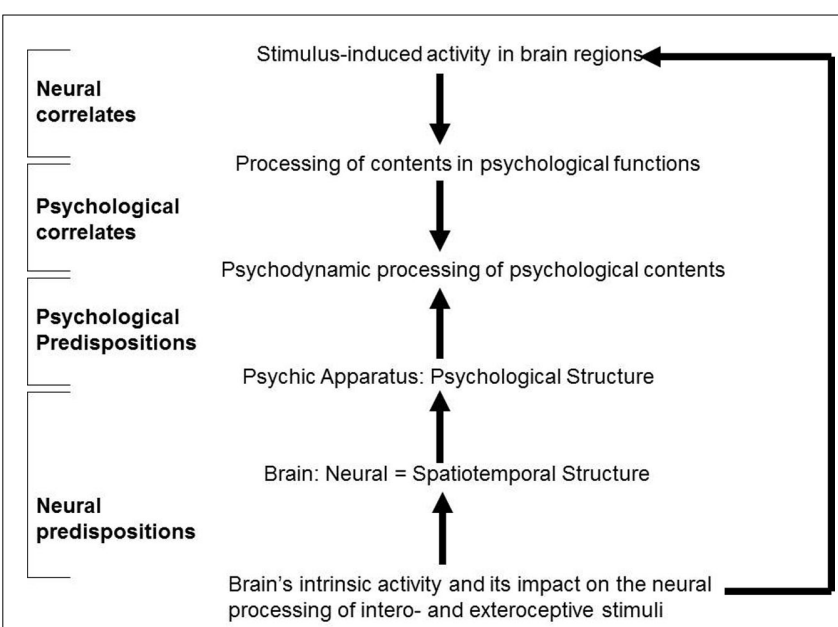

FIGURE 3 | "Neural correlates" versus "neural predispositions." 
statistically based spatiotemporal structure. Such spatiotemporal structure may be the neural predisposition not only for any kind of stimulus-induced activity but possibly also for the constitution of what Freud described as "psychological structure and organization" which he attributed to the psychic apparatus. And since the psychic apparatus' psychological structure is the psychological predisposition of various psychodynamic processes, the resting state's spatiotemporal structure may be (indirectly via the psychological structure) related and linked to the latter. We will then be able to develop what I described as the NPP and thereby to complement what Freud abandoned to do after 1895 (see Northoff, 2011 for details).

\section{IMPLICATIONS}

While the focus of this paper on Freud and the brain, I want to at least briefly indicate some of the implications of my resting statebased approach. For that I briefly discuss the examples of self/ego, dreams, cathexis, and depression.

The concepts of the self and ego are often taken in a substantial way as based on the concept of mind. Freud rejected such substance-based concept of the ego when is introduced his structural model. Empirically, such structural model implies that we may not be able to find a particular region to be specifically and exclusively associated with the functions of the ego and the ego itself Freud described. Instead of searching for the self-region or self-network in the brain, one may rather want to look for basic organizational and structural principles. More specifically, the here suggested spatiotemporal structure of the resting state may provide the starting point for such organizational principle. If being associated with the self or the ego, one would expect the organization of the resting state's spatiotemporal structure to somehow ingrain self-specific and thus to reflect the structure of the ego. This though would require much more extensive elaboration which is beyond the scope of this paper though (see Northoff, 2012).

Another examples are dreams. Dreams occur in the resting state activity of the brain. This raises the question how the thoughts, perceptions, and sense of self and ego are generated during the dream. Usually we associate those experiences with stimulusinduced activity. However, the fact that they can already occur in dreams indicates that the resting state activity by itself is principally able to generate dreams and ultimately consciousness. How is

\section{REFERENCES}

Arieli, A., Sterkin, A., Grinvald, A., and Aertsen, A. (1996). Dynamics of ongoing activity: explanation of the large variability in evoked cortical responses. Science 273, 1868-1871.

Beckmann, C., DeLuca, M., Devlin, J., and Smith, S. (2005). Investigations into resting-state connectivity using independent component analysis. Philos. Trans. R. Soc. Lond. B Biol. Sci. 360, 1001-1013.

Boly, M., Balteau, E., Schnakers, C., Degueldre, C., Moonen, G., Luxen, A., Phillips, C., Peigneux, P., Maquet, P., and Laureys, S. (2007). Baseline brain activity fluctuations

this possible? For that we need to shed a more detailed light on the specific organization of the resting state's spatiotemporal structure as indicated in the context of the self. More specifically, we may need to target the exact neuronal mechanisms of rest-rest interaction that seem to simulate some of the kind of neural activity that occurs during rest-stimulus interaction (see Northoff, 2011 for details). How such rest-rest and rest-stimulus interaction is related to consciousness remains unclear though at present (see Northoff, 2012).

Another interesting psychodynamic concept that appears in a new light is the one of cathexis. Freud introduced the term cathexis to describe psychic energy. The question is now whether there is a corresponding function and thus concept on the neuronal side. Lets recall. The brain spends $80 \%$ of its energy and metabolism already in the resting state while the stimulus-induced activity requires only small incremental increases in the energy supply. What does the brain do with all the energy? I here tentatively assumed that it constitutes a spatiotemporal structure. The exact neuronal mechanisms and its purpose remain unclear though (see Northoff, 2012). What is clear though is that the resting state may hold the key to find a neuronal analogon to what Freud described as cathexis on the psychological level.

Finally, one may want to point some clinical implication for depression. Depression can be characterized by abnormally high resting state activity in especially the midline regions (see Northoff, 2011). This may lead to an abnormally increased self-focus which in turn may abnormally filter all subsequent stimulus-induced activity and their related affective and cognitive functions. One question in this context may also be how the resting state's spatiotemporal structure and its alterations may be mirrored in and correspond to the changes in the spatial and temporal features of the patients' consciousness of themselves and the world including of time and space. That though requires a much more detailed phenomenological in-depth analysis and a corresponding neuroscientific investigation. While this is subject of future investigation, it makes clear that the resting state-based approach as proposed here leads to novel pathways in understanding (and also treating) psychiatric disorders like depression.

\section{ACKNOWLEDGMENTS}

I am grateful for financial support by CIHR, CIHR-EJLB, the Michael Smith Foundation, and HDRF/ISAN.

Buzsaki, G. (2006). Rhythms of the Brain. New York: Oxford University Press.

Buzsaki, G., and Draguhn, A. (2004). Neuronal oscillations in cortical networks. Science 304, 1926-1929.

Carhart-Harris, R. L., and Friston, K. J. (2010). The default-mode, egofunctions and free-energy: a neurobiological account of Freudian ideas. Brain 133, 1265-1283.

Damasio, A. R. (1995). Emotion, Reason and the Human brain. New York: Avon Books.

Damasio, A. R. (2000). The Feeling of What Happens. New York: Harvest Books.
Damoiseaux, J. S., Rombouts, S. A. R. B., Barkhof, F., Scheltens, P., Stam, C. J., Smith, S. M., and Beckmann, C. F. (2006). Consistent restingstate networks across healthy subjects. Proc. Natl. Acad. Sci. U.S.A. 103, 13848-13853.

Ellenberger, H. F. (1970). The Discovery of the Unconscious: The History and Evolution of Dynamic Psychiatry. New York: Basic Books.

Feinberg, T. E. (2009). From Axons to Identity: Neurological Explorations of the Nature of the Self. New York: W. W. Norton.

Feinberg, T. E. (2011). Neuropathologies of the self: clinical and 
anatomical features. Conscious. Cogn. 20, 75-81.

Fingelkurts, A. (2004). Making complexity simpler: multivariability and metastability in the brain. Int. J. Neurosci. 114, 843-862.

Fingelkurts, A., and Kahkonen, S. (2005). Functional connectivity in the brain - is it an elusive concept? Neurosci. Biobehav. Rev. 28, 827-836.

Fingelkurts, A. A., Fingelkurts, A. A., and Neves, C. E. H. (2010). Natural world physical, brain operational, and mind phenomenal space-time. Phys. Life Rev. 7, 195-249.

Fonagy, P. (2003). Psychoanalysis today. World Psychiatry 2, 73-80.

Fox, M., Snyder, A., Vincent, J., Corbetta, M., Van Essen, D., and Raichle, M. (2005). The human brain is intrinsically organized into dynamic, anticorrelated functional networks. Proc. Natl. Acad. Sci. U.S.A. 102, 9673-9678.

Fransson, P. (2005). Spontaneous lowfrequency BOLD signal fluctuations: an $\mathrm{AMRI}$ investigation of the restingstate default mode of brain function hypothesis. Hum. Brain Mapp. 26, 15-29.

Freeman, W. J. (2003). The wave packet: an action potential for the 21st century. J. Integr. Neurosci. 2, 3-30.

Freud, S. (1895). Project for a Scientific Psychology. London: Hogarth Press.

Freud, S. (1915). The Unconscious, 14th Edn. London: Hogarth Press.

Fries, P., Nikolic, D., and Singer, W. (2007). The gamma cycle. Trends Neurosci. 30, 309-316.

Fries, P., Reynolds, J., Rorie, A., and Desimone, R. (2001). Modulation of oscillatory neuronal synchronization by selective visual attention. Science 291, 1560-1563.

Gazzaniga, M. S., Ivry, R. B., and Mangun, G. R. (2008). Cognitive Neuroscience: The Biology of the Mind. New York: W. W. Norton.

Greicius, M., Srivastava, G., Reiss, A., and Menon, V. (2004). Defaultmode network activity distinguishes Alzheimer's disease from healthy aging: evidence from functional MRI. Proc. Natl. Acad. Sci. U.S.A. 101, 4637-4642.

He, B. J., and Raichle, M. E. (2009). The fMRI signal, slow cortical potential and consciousness. Trends Cogn. Sci. (Regul. Ed.) 13, 302-309.

He, B. J., Snyder, A. Z., Zempel, J. M., Smyth, M. D., and Raichle, M. E. (2008). Electrophysiological correlates of the brain's intrinsic large-scale functional architecture RID A-7795-2009. Proc. Natl. Acad. Sci. U.S.A. 105, 1603916044.
Hobson, J. A. (2009). REM sleep and dreaming: towards a theory of protoconsciousness. Nat. Rev. Neurosci. 10, 803-813.

Hunter, M. D., Eickhoff, S. B., Miller, T. W. R., Farrow, T. F. D., Wilkinson, I. D., and Woodruff, P. W. R. (2006). Neural activity in speech-sensitive auditory cortex during silence. Proc. Natl. Acad. Sci. U.S.A. 103, 189-194.

Kandel, E. (1998). A new intellectual framework for psychiatry. Am. J. Psychiatry 155, 457-469.

Khader, P., Schicke, T., Roeder, B., and Roesler, F. (2008). On the relationship between slow cortical potentials and BOLD signal changes in humans. Int. J. Psychophysiol. 67, 252-261.

Koch, C. (2004). The Quest for Consciousness: A Neurobiological Approach. Englewood: Roberts and Company Publishers.

Lashley, K. (1949). Persistent problems in the evolution of mind. Q. Rev Biol. 24, 28-48.

Levins, R. (2003). Whose scientific method? Scientific methods for a complex world. New Solut. 13, 261-274.

Lewis, C. M., Baldassarre, A., Committeri, G., Romani, G. L., and Corbetta, M. (2009). Learning sculpts the spontaneous activity of the resting human brain. Proc. Natl. Acad. Sci. U.S.A. 106, 17558-17563.

Llinas, R. (1988). The intrinsic electrophysiological properties of mammalian neurons - insights into central nervous-system function. Science 242, 1654-1664.

Logothetis, N. K., Murayama, Y., Augath, M., Steffen, T., Werner, J., and Oeltermann, A. (2009). How not to study spontaneous activity. $\mathrm{Neu}$ roimage 45, 1080-1089.

Maandag, N. J. G., Coman, D., Sanganahalli, B. G., Herman, P., Smith, A. J., Blumenfeld, H., Shulman, R. G., and Hyder, F. (2007). Energetics of neuronal signaling and fMRI activity. Proc. Natl. Acad. Sci. U.S.A. 104, 20546-20551.

Mancia, M. (2004). The dream between neuroscience and psychoanalysis. Arch. Ital. Biol. 142, 525-531.

Mancia, M. (2006a). Implicit memory and early unrepressed unconscious: their role in the therapeutic process (how the neurosciences can contribute to psychoanalysis). Int. J. Psychoanal. 87, 83-103.

Mancia, M. (2006b). Psychoanalysis and Neuroscience. Milan: Springer.

Morcom, A. M., and Flher, P. C. (2007). Does the brain have a baseline? Why we should be resisting a rest. $\mathrm{Neu}$ roimage $37,1073-1082$.
Muthukumaraswamy, S. D., Edden, R. A. E., Jones, D. K., Swettenham, J. B., and Singh, K. D. (2009). Resting GABA concentration predicts peak gamma frequency and fMRI amplitude in response to visual stimulation in humans. Proc. Natl. Acad. Sci. U.S.A. 106, 8356-8361.

Nase, G., Singer, W., Monyer, H., and Engel, A. K. (2003). Features of neuronal synchrony in mouse visual cortex. J. Neurophysiol. 90, 1115-1123.

Northoff, G. (2007). Psychopathology and pathophysiology of the self in depression - neuropsychiatric hypothesis. J. Affect. Disord. 104, 1-14.

Northoff, G. (2011). Neuropsychoanalysis in Practice: Brain, Self and Objects. New York: Oxford University Press.

Northoff, G. (2012). Unlocking the brain. Volume I: Coding. Volume II: Consicousness. New York: Oxford University Press.

Northoff, G., Bermpohl, F., Schoeneich, F., and Boeker, H. (2007a). How does our brain constitute defense mechanisms? First-person neuroscience and psychoanalysis. Psychother. Psychosom. 76, 141-153.

Northoff, G., Walter, M., Schulte, R. F., Beck, J., Dydak, U., Henning, A. Boeker, H., Grimm, S., and Boesiger, P. (2007b). GABA concentrations in the human anterior cingulate cortex predict negative BOLD responses in fMRI. Nat. Neurosci. 10, 1515-1517.

Northoff, G., and Boeker, H. (2006). Principles of neuronal integration and defense mechanisms: a neuropsychoanalytic hypothesis. Neuropsychoanalysis 8 69-84.

Northoff, G., Heinzel, A., Greck, M. Bennpohl, F., Dobrowolny, H., and Panksepp, J. (2006). Self-referential processing in our brain - a metaanalysis of imaging studies on the self. Neuroimage 31, 440-457.

Northoff, G., and Panksepp, J. (2008). The trans-species concept of self and the subcortical-cortical midline system. Trends Cogn. Sci. (Regul. Ed.) 12, 259-264.

Northoff, G., Wiebking, C., Feinberg, T., and Panksepp, J. (2011). The "resting-state hypothesis" of major depressive disorder-a translational subcortical-cortical framework for a system disorder. Neurosci. Biobehav. Rev. 35, 1929-1945.

Northoff, G., Qin, P., and Nakao, T. (2010). Rest-stimulus interaction in the brain: a review. Trends Neurosci. 33, 277-284.

Panksepp, J. (1998). Affective Neuroscience: The Foundations of Human and Animal Emotions. New York: Oxford University Press.
Panksepp, J., and Northoff, G. (2009). The trans-species core SELF: the emergence of active cultural and neuro-ecological agents through self-related processing within subcortical-cortical midline networks. Conscious. Cogn. 18, 193-215.

Peled, A. (2008). NeuroAnalysis: The Missing Link Between Psychoanalysis and Neuroscience. New York: Routledge.

Pugh, G. (2006). "Cooperation not incorporation: psychoanalysis and neuroscience," in Psychoanalysis and Neuroscience, ed. M. Mancia (Heidelberg: Springer), 33-62.

Pyka, M., Beckmann, C. F., Schoening, S., Hauke, S., Heider, D., Kugel, H., Arolt, V., and Konrad, C. (2009). Impact of working memory load on fMRI resting state pattern in subsequent resting phases. PLoS ONE 4, e7198. doi:10.1371/journal.pone.0007198

Raichle, M. E. (2009a). A brief history of human brain mapping. Trends Neurosci. 32, 118-126.

Raichle, M. E. (2009b). A paradigm shift in functional brain imaging. $J$. Neurosci. 29, 12729-12734.

Raichle, M. E. (2010). The brain's (dark energy). Sci. Am. 302, 44-49.

Raichle, M. E., MacLeod, A. M., Snyder, A. Z., Powers, W. J., Gusnard, D. A., and Shulman, G. L. (2001). A default mode of brain function. Proc. Natl. Acad. Sci. U.S.A. 98, 676-682.

Schneider, F., Bermpohl, F., Heinzel, A., Rotte, M., Walter, M., Tempelmann C., Wiebking, C., Dobrowolny, H., Heinze, H. J., and Northoff, G. (2008). The resting brain and our self: self-relatedness modulates resting state neural activity in cortical midline structures. Neuroscience 157, 120-131.

Schore, A. N. (2003). Affect Regulation and the Repair of the Self. New York: W. W. Norton.

Shulman, R. G., Hyder, F., and Rothman, D. L. (2009). Baseline brain energy supports the state of consciousness. Proc. Natl. Acad. Sci. U.S.A. 106, 11096-11101.

Shulman, R. G., Rothman, D. L., Behar, K. L., and Hyder, F. (2004). Energetic basis of brain activity: implications for neuroimaging. Trends Neurosci. 27, 489-495.

Simpson, J. R., Snyder, A. Z., Gusnard, D. A., and Raichle, M. E. (2001). Emotion-induced changes in human medial prefrontal cortex: I. During cognitive task performance. Proc. Natl. Acad. Sci. U.S.A. 98, 683-687.

Singer, W. (1999). Neuronal synchrony: a versatile code for the definition of relations? Neuron 24, 49-65, $111-125$. 
Solms, M. (1996). What are affects? Psyche (Stuttg.) 50, 485-522.

Solms, M. (1997). What is consciousness? J. Am. Psychoanal. Assoc. 45, 681-703; discussion 704-778.

Solms, M. (1999). The deep psychological functions of the right cerebral hemisphere. Inst. Psychoanal. Br. Psychoanal. Soc. 35, 9-29.

Solms, M. (2000). Dreaming and REM sleep are controlled by different brain mechanisms. Behav. Brain Sci. 23, 843-850; discussion 9041121.

Solms, M. (2004). Freud returns. Sci. Am. 290, 82-88.

Solms, M., and Solms-Kaplan, K. (2000). Clinical Studies in Neuro-
Psychoanalysis. London: Karnac Books.

Solms, M., and Turnbull, O. (2002). The Brain and the Inner World: An Introduction to the Neuroscience of Subjective Experience. New York: Other Press.

Solms, M., Turnbull, O. H., KaplanSolms, K., and Miller, P. (1998). Rotated drawing: the range of performance and anatomical correlates in a series of 16 patients. Brain Cogn. 38, 358-368.

Eijsden, P. V. A. N., Hyder, F., Rothman, D. L., and Shulman, R. G. (2009). Neurophysiology of functional imaging. Neuroimage 45, 1047-1054.
Wang, K., Liang, M., Wang, L., Tian, L., Zhang, X., Li, K., and Jiang, T. (2007). Altered functional connectivity in early Alzheimer's disease: a resting-state fMRI study. Hum. Brain Mapp. 28, 967-978.

Weinstein, E. A., and Kahn, R. L. (1956). Patterns of social interaction in brain disease. Am. J. Psychiatry $113,138-142$.

Conflict of Interest Statement: The author declares that the research was conducted in the absence of any commercial or financial relationships that could be construed as a potential conflict of interest.
Received: 18 November 2011; accepted: 24 February 2012; published online: 02 April 2012.

Citation: Northoff G (2012) Psychoanalysis and the brain - why did Freud abandon neuroscience? Front. Psychology 3:71. doi: 10.3389/fpsyg.2012.00071

This article was submitted to Frontiers in Psychoanalysis and Neuropsychoanalysis, a specialty of Frontiers in Psychology. Copyright ( 2012 Northoff. This is an open-access article distributed under the terms of the Creative Commons Attribution Non Commercial License, which permits non-commercial use, distribution, and reproduction in other forums, provided the original authors and source are credited. 